\title{
RADIAÇÃO GAMA NA CONSERVAÇÃO DE CAQUI 'GIOMBO', DESTANIZADO E FRIGOARMAZENADO' ${ }^{1}$
}

\author{
ROGÉRIO LOPES VIEITES², NAGELA FARIAS MAGAVE PICANÇO³ ${ }^{3}$ ÉRICA REGINA DAIUTO ${ }^{4}$
}

RESUMO-O caqui apresenta uma safra curta, sendo necessário estender seu período de comercialização utilizando técnicas adequadas de armazenamento. O objetivo deste trabalho foi avaliar o uso da radiação gama na qualidade pós-colheita de caquis ‘Giombo’ destanizados. Frutos colhidos meio-maduros e com aproximadamente $50 \%$ da coloração verde, foram destanizados, acondicionados em bandejas de poliestireno expandido (EPS), revestidas por filme plástico de polietileno de baixa densidade (PEBD) e submetidos à radiação gama $\left({ }^{60} \mathrm{Co}\right)$. Os frutos foram armazenados durante 35 dias, sob refrigeração $\left(0 \pm 0,5^{\circ} \mathrm{C}\right.$ e $\left.85 \pm 0,5 \% \mathrm{UR}\right)$. Os tratamentos realizados foram: T1 - 0,0 kGy; T2 - 0,3 kGy; T3 - 0,6 kGy; T4 - 0,9 kGy; T5 - 1,2 kGy. As análises realizadas a cada 7 dias foram: perda de massa, atividade respiratória, teores de sólidos solúveis (SS), ácido ascórbico, acidez titulável (AT) e relação SS/AT. Os frutos submetidos à dose de 0,6 kGy apresentaram o menor percentual de perda de massa e produção de $\mathrm{CO}_{2}$ ao longo do período experimental. Os teores de SS eAT permaneceram estáveis e sem diferenças devido às doses de irradiação aplicadas. Os tratamentos com 0,3 KGy e 0,6 KGy foram os mais eficazes na manutenção da firmeza nos caquis. Nos teores de ácido ascórbico, observou-se redução ao longo do período experimental, sendo os menores valores apresentados para a maior dose de irradiação (1,2 KGy).

Termos de indexação: Diospyrus kaki, pós-colheita, refrigeração, adstringência.

\section{GAMMA RADIATION IN THE CONSERVATION OF 'GIOMBO’PERSIMMON, WITHOUT ADSTRINGENCY STORED UNDER REFRIGERATION}

\begin{abstract}
The persimmon presents a short harvest, being necessary to extend his commercialization period using appropriate storage techniques. The purpose of this work was to evaluate the use of the gamma radiation in the postharvest quality 'Giombo' persimmons without adstringency. Fruits were harvest middleripe, with approximately $50 \%$ of the green coloration and were submitted to the process of astringency removal, packed in expanded polystyrene (EPS) trays, covered by low densidy polyethylene (LDPE) plastic film and submitted the gamma radiation $\left({ }^{60} \mathrm{Co}\right)$. The fruits were stored for 35 days, under refrigeration $(0 \pm 0,5$ ${ }^{\circ} \mathrm{C}$ and $85 \pm 0,5 \%$ of relative humidity). The evaluated treatments were: T1 - $0.0 \mathrm{kGy}$; T2 - $0.3 \mathrm{kGy}$; T3 $0.6 \mathrm{kGy}$; T4 - 0.9 kGy; T5 - $1.2 \mathrm{kGy}$. The evaluated analyses every 7 days were: weight loss, respiratory activity, soluble solids (SS), ascorbic acid content, acidity titulável (AT), and relation SS/AT. The fruits submitted to the $0.6 \mathrm{kGy}$ presented the percentile smallest of weight loss and smaller $\mathrm{CO}_{2}$ production along the experimental period. The SS and AT content stayed stable and without difference among the irradiation applied. The treatments with $0.3 \mathrm{kGy}$ e $0.6 \mathrm{kGy}$ were the most effective in the maintenance of the persimmon firmness values. For the ascorbic acid content was observed reduction along the experimental period being the smallest values presented for the largest irradiation dose (1,2 KGy).
\end{abstract}

Index terms: Diospyrus kaki, postharvest, refrigeration, adstringency.

\footnotetext{
${ }^{1}$ (Trabalho 047-12). Recebido em: 04-01-2012. Aceito para publicação em: 28-08-2012. Projeto Financiado pelo CNPQ.

${ }^{2}$ Prof. Titular,docente Departamentos de Gestão e Tecnologia Agroindustrial da Faculdade de Ciências Agronômicas da UNESP-

-Botucatu. E mail: vieites@fca.unesp.br

${ }^{3}$ Doutora pelo curso Energia na Agricultura Faculdade de Ciências Agronômicas da UNESP-Botucatu .

${ }^{4}$ Pós doutoranda no Curso Horticultura da Faculdade de Ciências Agronômicas da UNESP-Botucatu. Email: erdaiuto@uol.com.br

${ }^{5}$ Mestranda FCA/UNESP-Botucatu- Bolsista FAPES.
} 


\section{INTRODUÇÃO}

O caqui tem excelente sabor, aparência atraente e boa qualidade nutricional, além de ser fonte de fibras, carboidratos, vitaminas e sais minerais, o que garante boa aceitação deste no mercado. No entanto, sua colheita está concentrada em três meses do ano, sendo a falta de informações consistentes quanto ao armazenamento e transporte fatores limitantes à expansão da cultura, acarretando perdas no final do processo produtivo, bem como durante a comercialização do fruto (DONAZZOLO; BRACKMANN, 2002).

$O$ caqui 'Giombo' pertence ao grupo denominado "variável" e inclui frutos de polpa taninosa e de cor amarelada, quando sem sementes e não taninosa, parcial ou totalmente, quando com uma ou mais sementes (CAMPO-DALL'ORTO et al.,1996). Na ausência de sementes, os frutos preservam a adstringência mesmo quando amadurecidos, necessitando de processo artificial para sua remoção. Este processo é denominado destanização. O maior inconveniente de se acelerar o processo de amadurecimento, para promover a destanização dos frutos, é a diminuição de sua vida de prateleira (EDAGI ; KLUGE, 2009). Geralmente, frutos de caqui ‘Giombo' são expostos ao vapor do álcool etílico, que é um método prático de destanização, mas, segundo Antoniolli et al. (2000), o processo pode comprometer a firmeza da polpa, quando armazenado por períodos prolongados.

A utilização de práticas que atrasem a colheita dos frutos e prolonguem o armazenamento, podem ser uma alternativa para aumentar a rentabilidade da cultura. Dentre estas práticas, tem-se a utilização de armazenamento refrigerado, que mantém a qualidade dos frutos por um curto espaço de tempo (CHITARRA; CHITARRA, 2005). Segundo Turk (1993), o armazenamento de caqui entre 0 e $2^{\circ} \mathrm{C}$, com 90\% UR, não passa de 2 ou 3 meses. Aliada ao armazenamento em baixa temperatura, podese utilizar atmosfera controlada para prolongar o armazenamento dos frutos, diminuindo as perdas qualitativas, o que permite manter a qualidade do caqui por mais de 3 meses (VIDRIH et al., 1990). $\mathrm{O}$ uso de cera e de atmosfera modificada pode prolongar a vida útil de caquis ‘Giombo' por 6 dias (BLUM et al., 2008). Brackmann et al. (2003) também avaliaram o efeito do 1-MCP aplicado em diferentes épocas, durante o armazenamento refrigerado, em caqui cv. Quioto, visando a inibir o efeito do etileno e estendendo o tempo e a qualidade dos frutos armazenados. A literatura não aborda o uso de radiação gama na conservação de caquis.

Associada aos procedimentos pós-colheita normalmente empregados, as radiações gama, em baixos níveis de dose, são um excelente método para prolongar a vida comercial das frutas, retardando os processos de amadurecimento e senescência, bem como reduzindo significativamente o apodrecimento causado por fungos e bactérias patogênicas (KÄFERSTEIN; MOY, 1993).

Objetivou-se avaliar o efeito da radiação gama na qualidade pós-colheita de caquis 'Giombo' destanizados.

\section{MATERIAL E MÉTODOS}

Foram utilizados caquis 'Giombo', safra de 2009, adquiridos na Fazenda Sacramento Agropastoril Ltda,pertencente ao grupo Sanhaço, cidade de Avaré (SP), localizada à latitude $23^{\circ} 05^{\prime} 56^{\prime \prime}$, longitude $48^{\circ} 55^{\prime} 33^{\prime \prime} \mathrm{W}$ e altitude de 780 metros, com precipitação anual de 1.500 a $1.700 \mathrm{~mm}$ ano-1, temperatura anual entre $20-24$ ${ }^{\circ} \mathrm{C}$ e solo classificado como Latossolo Roxo (terra roxa estruturada, terra roxa latossólica). Os frutos colhidos foram selecionados quanto ao estádio de maturação, sendo classificados visualmente quanto à coloração em estádio 3 , fruto meio-maduro com aproximadamente 50\% da coloração verde (Programa Brasileiro para a Modernização da Horticultura, 2000). Os frutos foram destanizados, sendo este procedimento efetuado com álcool etílico. Os frutos foram colocados em caixas de plástico, contendo cerca de 10 kg e levados à câmara de refrigeração. Na câmara de refrigeração, estas caixas foram cobertas com lona e instalou-se o destanizador, utilizado como recipiente do álcool etílico. Utilizaram-se $80 \mathrm{~mL}$ de álcool etílico para cada $10 \mathrm{~kg}$ de fruta, em um período de $24 \mathrm{~h}$, a $26{ }^{\circ} \mathrm{C}$. No destanizador, foram utilizadas 2 lâmpadas de $150 \mathrm{~W}$ cada, que aqueciam o álcool, favorecendo sua evaporação. A seguir, os frutos foram acondicionados em bandejas de poliestireno expandido, com duas unidades, e embalados com filme de policloreto de vinila com 0,020 mm. Após a montagem dos experimentos, os frutos foram armazenados em câmara fria a $4{ }^{\circ} \mathrm{C}$, durante 12 horas. Em seguida, foram transportados, sem refrigeração, à C.B.E. (Companhia Brasileira de Esterilização), localizada na Rodovia Dom Pedro I, Km 89,5, Ponte Alta, Jarinu - SP, onde receberam a aplicação de radiação ionizante (raios gama ${ }^{60} \mathrm{Co}$ ). Foi utilizado como dosímetro o Gammachrome (Harwell Dosimeters).

Os frutos de caqui destanizados foram submetidos a quatro doses de radiação. Os tratamentos foram: T1 - 0,0 kGy; T2 - 0,3 kGy; 
T3 - 0.6 kGy; T4 - 0.9 kGy; T5 - 1,2 kGy.Os frutos foram armazenados a $0 \pm 0,5^{\circ} \mathrm{C}, 85 \pm 0,5 \%$ UR, por 35 dias. As análises realizadas a cada 7 dias foram: perda de massa, atividade respiratória, firmeza, teores de sólidos solúveis (SS), acidez titulável (AT), ácido ascórbico, assim como a relação SS/AT. As análises foram realizadas em triplicata.

A perda de massa fresca foi obtida pela pesagem das embalagens em balança analítica, considerando a massa inicial de cada amostra, com os resultados expressos em percentagem.

A atividade respiratória foi determinada pela liberação de $\mathrm{CO}_{2}$ em cada embalagem, de acordo com metodologia adaptada de Bleinroth et al. (1976), utilizando-se para isso de solução de hidróxido de bário saturado e solução de hidróxido de potássio $0,1 \mathrm{~N}$. Para tanto, foi utilizada a seguinte fórmula: $\mathrm{TCO}_{2}=2,2$ (Vo-V1). 10/P.T. Em que: $\mathrm{T}$ $\mathrm{CO}_{2}=$ taxa de respiração $\left(\mathrm{mL}\right.$ de $\left.\mathrm{CO}_{2} \cdot \mathrm{Kg}^{-1} \cdot \mathrm{h}^{-1}\right)$; Vo = volume gasto de $\mathrm{HCl}$ para titulação de hidróxido de potássio - padrão antes da absorção de $\mathrm{CO}_{2}(\mathrm{~mL})$; V1 = volume gasto de ácido clorídrico $(\mathrm{HCl})$ para titulação de hidróxido de potássio após a absorção de $\mathrm{CO}_{2}$ da respiração $(\mathrm{mL}) ; \mathrm{P}=$ massa dos frutos; $\mathrm{T}$ = tempo da respiração; 2,2 = inerente ao equivalente de $\mathrm{CO}_{2}$ (44/2), multiplicado pela concentração do ácido clorídrico; 10 = ajuste para o total de hidróxido de potássio utilizado.

O teor de sólidos solúveis foi realizado através de leitura refratométrica ( $\left.{ }^{\circ} \mathrm{Brix}\right)$, em três amostras, utilizou-se refratômetro tipo Abbe, marca ATAGO - N.

A acidez titulável (AT) foi expressa em g de ácido málico $100 \mathrm{~g}^{-1}$, determinado em três amostras, com titulação de 10 gramas de polpa homogeneizada diluída para $100 \mathrm{~mL}$ com água destilada, com solução padronizada de hidróxido de sódio a $0,1 \mathrm{M}$, tendo como indicador o Instituto Adolfo Lutz, 2008.

A firmeza foi determinada com auxílio do Texturômetro (STEVENS - LFRA texture analyser) com a distância de penetração de $10 \mathrm{~mm}$ e velocidade de 2,0 $\mathrm{mm} \mathrm{seg}^{-1}$, utilizando-se do ponteiro TA 9/1000. $\mathrm{O}$ valor obtido para se determinar a textura em gramas/força por centímetro quadrado $\left(\mathrm{gf} / \mathrm{cm}^{2}\right)$ é definido como a força requerida para que uma parte do ponteiro penetre na polpa do produto. Foram realizadas 5 leituras para cada uma das 3 repetições, em todos os tratamentos.

Para a determinação do teor de ácido ascórbico, os extratos foram obtidos com adição de $30 \mathrm{~mL}$ de ácido oxálico a $30 \mathrm{~g}$ de polpa, que foram congelados em seguida. O conteúdo de ácido ascórbico foi determinado a partir de $10 \mathrm{~g}$ da polpa, por titulação em ácido oxálico a 0,5\%, com 2,6 diclorofenolindofenol de sódio a 0,01M, com resultados expressos em $\mathrm{mL}$ de ácido ascórbico $100 \mathrm{~mL}^{-1}$ de polpa (MAPA, 2006).

$\mathrm{O}$ delineamento experimental empregado foi o inteiramente casualizado (DIC), em fatorial 5 x 6 (irradiação x tempo), no segundo experimento. A comparação entre as médias foi feita utilizandose do teste de Tukey, a 5\% de probabilidade (GOMES, 1987).

\section{RESULTADOS E DISCUSSÃO}

A perda de massa fresca foi crescente nos frutos de todos os tratamentos durante o período experimental (Figura 1). Para a maioria dos produtos hortícolas frescos, a máxima perda de massa fresca tolerada para o não aparecimento de murcha e/ou enrugamento da superfície oscila entre 5 e 10\% (FINGER ; VIEIRA, 2002). Observou-se, neste trabalho, que a perda de massa permaneceu numa faixa aceitável, e os caquis de todos tratamentos, inclusive os do controle, ainda se apresentavam viáveis para a comercialização. Este resultado encontrado se deve ao fato de o armazenamento ser realizado sob refrigeração, o que já minimiza a perda de peso devido o efeito da respiração e à transpiração.

Os resultados também mostram o efeito benéfico da aplicação da radiação gama nos caquis. Frutos submetidos à dose de 0,6 kGy apresentaram o menor percentual de perda de massa, enquanto os frutos-controle e os submetidos à maior dose de irradiação (1,2 kGy) apresentaram os maiores percentuais de perda de massa durante o armazenamento (2,79\% e 2,59\%, respectivamente). Pressupõe-se que doses mais elevadas de irradiação promovam maior perda de massa nos caquis, pois Maxie et al. (1971) relataram que a irradiação pode aumentar a permeabilidade de membranas, incrementar a atividade metabólica e romper ligações intercelulares, além de aumentar a transpiração através da cutícula, aumentando a perda de massa, quando em doses mais elevadas.

Para a atividade respiratória, observouse que os frutos apresentaram redução na produção de $\mathrm{CO}_{2}$ logo no dia 7 de armazenamento (Figura 2). Segundo Chitarra e Chitarra (2005), as baixas temperaturas retardam e diminuem a intensidade do pico climatério, reduzindo a respiração e o consequente consumo de reservas. Para Hardenburg et al. (1986), o caqui apresenta baixa taxa de respiração (2 a $5 \mathrm{mg}$ de $\mathrm{CO}_{2} \mathrm{~kg}^{-1} \mathrm{~h}^{-1} 1$, a $0{ }^{\circ} \mathrm{C} \mathrm{e}, 30$ a $40 \mathrm{mg} \mathrm{CO}_{2} \cdot \mathrm{kg}^{-1} \mathrm{~h}^{-1}$ a $25^{\circ} \mathrm{C}$ ).

A partir do $14^{\circ}$ dia de armazenamento, 
observou-se aumento na taxa respiratória com maior produção de $\mathrm{CO}_{2}$ nos frutos do $\mathrm{T} 1$, seguido dos frutos do T5 e T4, com 0,95; 0,78 e 066 $\mathrm{mL} \mathrm{CO} \mathrm{kg} \mathrm{h}^{-1}$. No $21^{\circ}$ dia de armazenamento, observou-se, nos frutos do T1, declínio na taxa respiratória, enquanto nos frutos do T3, T4 e T5, a tendência de produção de $\mathrm{CO}_{2}$ foi linear. No final do período de armazenamento, verificou-se a maior produção de $\mathrm{CO}_{2}$ nos frutos do T4 e T5, com 1,82 e 1,15 $\mathrm{mL} \mathrm{CO}_{2} \mathrm{~kg} \mathrm{~h}^{-1}$, respectivamente. Os tratamentos com as menores doses de irradiação, ou seja, 0,3 e 0,6 kGy, apresentam menor intensidade respiratória durante o período experimental. Portanto, da mesma forma que ocorreu para perda de massa. As doses menores foram mais efetivas na conservação dos caquis.

Os teores SS variaram de 15 a $21^{\circ}$ Brix (Tabela 1). Verificou-se ligeiro aumento nos teores de SS no $21^{\circ}$ dia no T1, com declínio desses valores nos dias subsequentes. Nos demais tratamentos, não foram verificadas diferenças significativas nos teores de SS, com exceção do $14^{\circ}$ dia do T5. Os sólidos solúveis apresentam tendência de aumento com o amadurecimento devido ao aumento no teor de açúcares simples (CHITARRA ; CHITARRA, 2005). Silva (2008) não observou diferença significativa entre os tratamentos e dias de armazenamento em caqui "Fuyu", para este parâmetro.

A AT variou de 0,8 a $1,2 \%$ de ácido málico durante o período experimental. Valores semelhantes foram encontrados por Fagundes (2004), entre 0,08 e 0,10\% de ácido málico, para o caqui 'Fuyu'.

Assim como para os teores de SS, observou-se estabilidade nos teores de AT, nos frutos de todos os tratamentos. Na maioria dos frutos, durante a maturação, ocorre diminuição na AT devido ao aproveitamento de ácidos como fonte de energia (WILLS et al., 1981). Em caquis, a AT também foi decrescente durante o processo de amadurecimento (DAOOD et al., 1992), mas, segundo Senter et al. (1991), ela não varia significativamente durante o amadurecimento do caqui.

Para SS e AT, não foram observadas diferenças entre as doses de irradiação. $O$ fato de o caqui ser um fruto climatérico de baixa intensidade respiratória, associado ao armazenamento refrigerado e atmosfera modificada passiva, refletiu nos resultados de SS e AT, tendo como consequência, a estabilidade destes parâmetros ao longo do período experimental.
Para a variável ratio, que está relacionada ao índice de maturação dos frutos, observou-se diferença estatística apenas nos frutos T1, no $28^{\circ}$ dia, com a menor média. Com relação ao período de armazenamento, foi verificado que os frutos de $\mathrm{T} 2$, T4 e T5 apresentaram as maiores médias no final do experimento, em função da redução da AT, pois os teores de SS permaneceram estáveis. Observouse que, nos frutos de T1 e T3, houve redução do ratio, provavelmente devido à amostragem, pois o método de análise foi destrutivo, de forma que os frutos comparados podem apresentar teores iniciais diferenciados.

A firmeza dos caquis não foi influenciada pelos tratamentos até o $14^{\circ}$ dia de armazenamento. No $21^{\circ}$ e $28^{\circ}$ dia, observou-se que os frutos do tratamento-controle apresentavam a menor média, diferindo dos demais tratamentos e pressupondo-se efeito benéfico da radiação gama nos frutos. Essa redução nos valores de firmeza, provavelmente, ocorreu devido ao processo de amadurecimentos dos frutos do controle. Entretanto, no final do período de armazenamento, verificou-se que, para o tratamento-controle, o valor da firmeza dos frutos foi maior que na análise anterior. Isto pode ser justificado em função da metodologia utilizada, em que os frutos comparados podem apresentar teores iniciais diferenciados e, além disto, alguns frutos apresentavam processo de amadurecimento avançado.

Nos frutos irradiados, observou-se, ao final do armazenamento, maiores valores de firmeza para as doses 0,3 e 0,6 kGy. Portanto, estas dosagens foram mais eficazes para a manutenção deste parâmetro nos caquis, evidenciando que a irradiação em doses menores pode contribuir para a manutenção da firmeza dos frutos.

Os teores de ácido ascórbico diminuíram significativamente nos frutos de todos os tratamentos (Tabela 3), o que também foi observado por Blum et al. (2008) em caquis 'Giombo' submetidos a tratamento com cera durante o armazenamento, e por Antoniolli et al. (2000) em frutos da mesma cultivar.

O período de armazenamento influenciou de forma significativa na redução dos teores de ácido ascórbico dos caquis submetidos a 1,2 kGy, sendo observado os menores valores para este parâmetro em comparação aos demais tratamentos. Assim como observado nesta pesquisa, Kovacs et al. (1994), pesquisando as mudanças nos ácidos orgânicos em maçã irradiada, nas doses de 0,5 kGy a 5,0 kGy, verificaram que, após 21 dias, os conteúdos dos ácidos málico e ascórbico diminuíram com o aumento dos níveis de irradiação. 


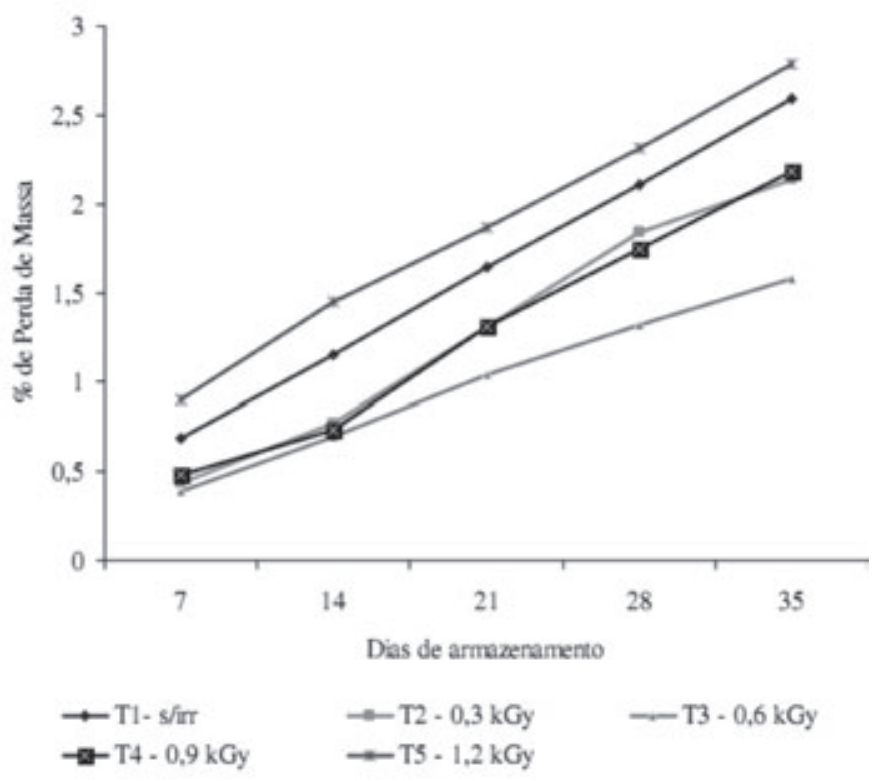

FIGURA 1- Perda de massa por caquis 'Giombo', colhidos no estádio 3 de maturação (“meio maduro”), destanizados, irradiados e armazenados a $0{ }^{\circ} \mathrm{C}$ e $85 \%$ UR.

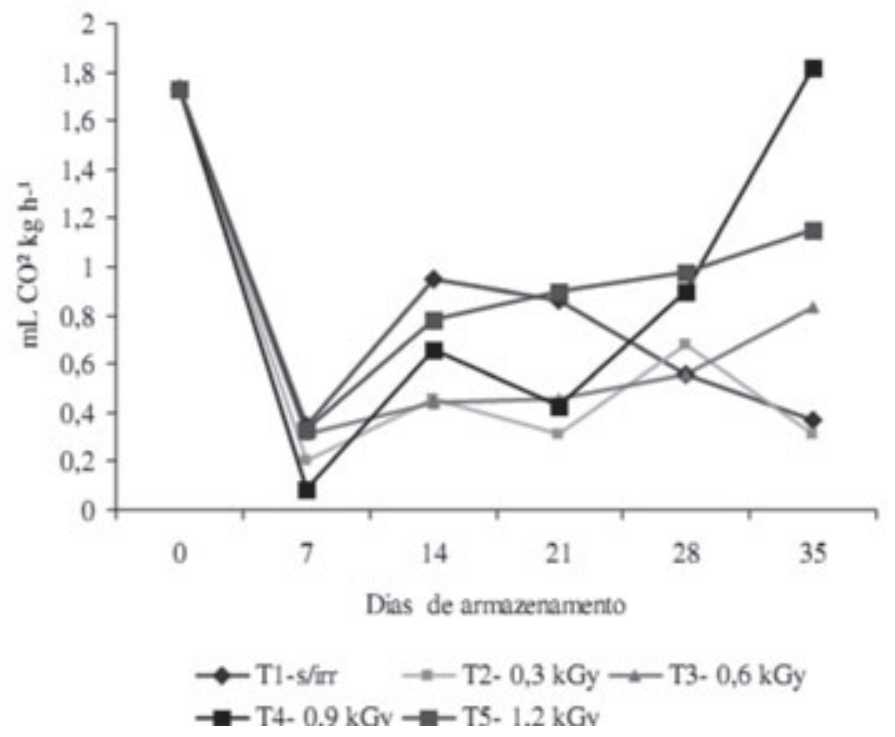

FIGURA 2- Atividade respiratória ( $\mathrm{mL}$ de $\left.\mathrm{CO}_{2} \mathrm{~kg} / \mathrm{h}^{-1}\right)$ de caquis 'Giombo', colhidos no estádio 3 de maturação (“meio maduro”), destanizados, irradiados e armazenados a $0{ }^{\circ} \mathrm{C}$ e $85 \%$ UR. 
TABELA1-Teores de sólidos solúveis, acidez titulável, assim como a relação sólidos solúveis/acidez titulável em caquis ‘Giombo', colhidos no estádio 3 de maturação (“meio maduro”), destanizados, irradiados e armazenados a $0{ }^{\circ} \mathrm{C}$ e $85 \%$ UR.

\begin{tabular}{|c|c|c|c|c|c|c|}
\hline \multirow{2}{*}{$\begin{array}{c}\text { Tratamento } \\
\text { (temperatura) }\end{array}$} & \multicolumn{6}{|c|}{ Dias de análise } \\
\hline & $\mathbf{0}$ & 7 & 14 & 21 & 28 & 35 \\
\hline & \multicolumn{6}{|c|}{ Sólidos solúveis ( ${ }^{\circ}$ Brix) } \\
\hline $\mathrm{T} 1=0,0 \mathrm{kGy}$ & 19,33aA & $18,67 \mathrm{aAB}$ & $21,00 \mathrm{aA}$ & $20,00 \mathrm{aAB}$ & $18,00 \mathrm{aB}$ & $20,00 \mathrm{aAB}$ \\
\hline $\mathrm{T} 2=0,3 \mathrm{kGy}$ & 19,33aA & 18,67aA & 19,33aA & $18,67 \mathrm{aA}$ & $18,67 \mathrm{aA}$ & 20,33aA \\
\hline $\mathrm{T} 3=0,6 \mathrm{kGy}$ & 19,33aA & 19,33aA & $20,67 \mathrm{aA}$ & $20,00 \mathrm{aA}$ & $17,00 \mathrm{aA}$ & 19,00aA \\
\hline $\mathrm{T} 4=0,9 \mathrm{kGy}$ & $19,33 a A$ & 17,33aA & $19,67 \mathrm{aA}$ & $19,67 \mathrm{aA}$ & $17,00 \mathrm{aA}$ & 19,00aA \\
\hline $\mathrm{T} 5=1,2 \mathrm{kGy}$ & 19,33aA & $17,67 \mathrm{aB}$ & $15,00 \mathrm{bB}$ & $20,33 a A$ & $19,00 \mathrm{aA}$ & 18,33aA \\
\hline \multicolumn{7}{|l|}{ CV (\%) 16,71} \\
\hline & \multicolumn{6}{|c|}{ Acidez titulável (g.ác.málico 100g de polpa-1) } \\
\hline $\mathrm{T} 1=0,0 \mathrm{kGy}$ & $0,09 \mathrm{aA}$ & $0,10 \mathrm{aA}$ & $0,12 \mathrm{aA}$ & $0,12 \mathrm{aA}$ & $0,11 \mathrm{aA}$ & $0,10 \mathrm{aA}$ \\
\hline $\mathrm{T} 2=0,3 \mathrm{kGy}$ & $0,09 \mathrm{aA}$ & $0,08 \mathrm{aA}$ & 0,09abA & $0,08 \mathrm{bA}$ & $0,07 \mathrm{bA}$ & 0,09aA \\
\hline $\mathrm{T} 3=0,6 \mathrm{kGy}$ & 0,09aA & $0,10 \mathrm{aA}$ & $0,12 \mathrm{aA}$ & $0,10 \mathrm{abA}$ & 0,10abA & $0,10 \mathrm{aA}$ \\
\hline $\mathrm{T} 4=0,9 \mathrm{kGy}$ & 0,09aA & $0,09 \mathrm{aA}$ & 0,08abA & 0,09abA & $0,07 \mathrm{bA}$ & $0,08 \mathrm{aA}$ \\
\hline $\mathrm{T} 5=1,2 \mathrm{kGy}$ & 0,09aA & $0,09 \mathrm{aA}$ & $0,07 \mathrm{bA}$ & 0,09abA & 0,09abA & 0,09aA \\
\hline \multicolumn{7}{|l|}{ CV (\%) 18,50} \\
\hline & \multicolumn{6}{|c|}{ Sólidos solúveis/acidez titulável } \\
\hline $\mathrm{T} 1=0,0 \mathrm{kGy}$ & $222,87 a A$ & $192,43 a A$ & $110,63 a A$ & $167,37 a A$ & $163,37 \mathrm{bA}$ & $185,10 \mathrm{aA}$ \\
\hline $\mathrm{T} 2=0,3 \mathrm{kGy}$ & $222,87 \mathrm{aA}$ & $248,07 \mathrm{aA}$ & $220,10 \mathrm{aA}$ & $234,90 \mathrm{aA}$ & 252,93aA & $226,03 a A$ \\
\hline $\mathrm{T} 3=0,6 \mathrm{kGy}$ & $222,87 \mathrm{aA}$ & $193,67 \mathrm{aA}$ & $182,77 \mathrm{aA}$ & 199,33aA & 206,83abA & $192,43 a A$ \\
\hline $\mathrm{T} 4=0,9 \mathrm{kGy}$ & $222,87 \mathrm{aA}$ & $200,00 \mathrm{aA}$ & 238,63aA & $210,90 \mathrm{aA}$ & 246,80aA & $234,06 \mathrm{aA}$ \\
\hline $\mathrm{T} 5=1,2 \mathrm{kGy}$ & $222,87 a A$ & 201,83aA & $202,67 \mathrm{aA}$ & $216,00 \mathrm{aA}$ & 206,53abA & 211,63aA \\
\hline CV (\%) 16,71 & & & & & & \\
\hline
\end{tabular}

Médias seguidas da mesma letra, minúscula na coluna e maiúscula na linha, não diferem entre si, pelo teste de Tukey, a 5\% de probabilidade.

TABELA 2- Firmeza de caquis 'Giombo', colhidos no estádio 3 de maturação (“meio maduro”), destanizados, irradiados e armazenados a $0{ }^{\circ} \mathrm{C}$ e $85 \%$ UR.

\begin{tabular}{|c|c|c|c|}
\hline $\begin{array}{c}\text { Tratamento } \\
\text { (temperatura) }\end{array}$ & & Dias de análise & \\
\hline & 7 & 21 & 28 \\
\hline & & Firmeza $\left(\mathrm{gf} \mathrm{cm}^{-2}\right)$ & \\
\hline $\mathrm{T} 1=0,0 \mathrm{kGy}$ & $238,67 \mathrm{aA} \quad 171,33 \mathrm{aAB}$ & 263,77aAB 85,33bAB & $36,00 \mathrm{bB} \quad 261,00 \mathrm{abA}$ \\
\hline $\mathrm{T} 2=0,3 \mathrm{kGy}$ & 238,67aA 298,33аA & $252,67 a A B \quad 334,67 a A$ & $337,00 \mathrm{aA} \quad 312,00 \mathrm{aA}$ \\
\hline T3= 0,6 kGy & $238,67 a A \quad 257,33 a A$ & $210,00 \mathrm{aA} \quad 264,00 \mathrm{aA}$ & 163,67abA 301,33aA \\
\hline $\mathrm{T} 4=0,9 \mathrm{kGy}$ & $238,67 \mathrm{aA} \quad 185,67 \mathrm{aA}$ & 175,33aA 244,00abA & 260,00aA 179,33abA \\
\hline T5= 1,2 kGy & 238,67aA $167,33 a A$ & $156,67 \mathrm{aA} \quad 202,00 \mathrm{abA}$ & $233,00 \mathrm{aA} \quad 109,67 \mathrm{bA}$ \\
\hline CV (\%) 36,10 & & & \\
\hline
\end{tabular}

Médias seguidas da mesma letra, minúscula na coluna e maiúscula na linha, não diferem entre si, pelo teste de Tukey, a 5\% de probabilidade. 
TABELA 3 - Teores de ácido ascórbico em caquis ‘Giombo’, colhidos no estádio 3 de maturação (“meio maduro"), destanizados, irradiados e armazenados a $0{ }^{\circ} \mathrm{C}$ e $85 \% \mathrm{UR}$.

\begin{tabular}{|c|c|c|c|c|c|c|}
\hline \multirow[t]{2}{*}{$\begin{array}{c}\text { Tratamento } \\
\text { (temperatura) }\end{array}$} & \multicolumn{6}{|c|}{ Dias de análise } \\
\hline & 0 & 7 & 14 & 21 & 28 & 35 \\
\hline & Acido & Iscórbico & (mL á & . Ascó & jico 1 & $\overline{0 m L-1)}$ \\
\hline $\mathrm{T} 1=0,0 \mathrm{kGy}$ & $5,00 \mathrm{aA}$ & 4,33abA & $2,33 \mathrm{aA}$ & $2,00 \mathrm{a} A$ & $2,00 \mathrm{aA}$ & $1,67 a A$ \\
\hline $\mathrm{T} 2=0,3 \mathrm{kGy}$ & $5,00 \mathrm{aA}$ & $3,00 \mathrm{bA}$ & $2,67 a A$ & $1,67 \mathrm{a} A$ & $1,67 \mathrm{aA}$ & $1,67 \mathrm{aA}$ \\
\hline $\mathrm{T} 3=0,6 \mathrm{kGy}$ & $5,00 \mathrm{aA}$ & 3,33abA & $2,00 \mathrm{aA}$ & $2,00 \mathrm{a} A$ & $2,00 \mathrm{aA}$ & $2,00 \mathrm{aA}$ \\
\hline $\mathrm{T} 4=0,9 \mathrm{kGy}$ & $5,00 \mathrm{aA}$ & $2,00 \mathrm{bA}$ & $2,00 \mathrm{aA}$ & $1,67 \mathrm{a} A$ & $2,00 \mathrm{aA}$ & $1,67 \mathrm{aA}$ \\
\hline $\begin{array}{c}\mathrm{T} 5=1,2 \mathrm{kGy} \\
\mathrm{CV}(\%) 28,00\end{array}$ & $5,00 \mathrm{aA}$ & $5,50 \mathrm{aA}$ & $2,33 \mathrm{aB}$ & $2,00 \mathrm{aF}$ & $2,67 \mathrm{aB}$ & $1,33 \mathrm{aC}$ \\
\hline
\end{tabular}

Médias seguidas da mesma letra, minúscula na coluna e maiúscula na linha, não diferem entre si, pelo teste de Tukey, a 5\% de probabilidade.

\section{CONCLUSÃO}

Frutos de caqui 'Giombo' destanizados, armazenados sob refrigeração e submetidos à radiação gama, na dose de entre 0,3 e 0,6 kGy, apresentaram os menores valores de perda de massa e produção de $\mathrm{CO}_{2}$, além da manutenção nos valores de firmeza.

\section{AGRADECIMENTOS}

Ao Conselho Nacional de Desenvolvimento Científico e Tecnológico.

\section{REFERÊNCIAS}

ANTONIOLLI, L.R; CAMARGO e CASTRO, P. R.; KLUGE, R. A.; SCARPARE, F. J. A. Remoção da adstringência de frutos de caquizeiro 'Giombo' sob diferentes períodos de exposição ao vapor de álcool etílico. PesquisaAgropecuária Brasileira, Brasília, v.35, n.10, p.2.083-2091, 2000.

BLEINROTH, E.W.; ZUCHINI, A.G.; POMPEO, R.M. Determinação das características físicas e mecânicas de variedade de abacate e sua conservação pelo frio. Coletânea ITAL, Campinas, v.7, n.1, p.29-81, 1976.

BLUM, J.;AYUB, A.; MALGARIM, M.B. Época de colheita e qualidade pós-colheita do caqui cv. Fuyu com a aplicação pré-colheita de ácido giberélico e Aminoetoxivinilglicina. Revista Biotemas, Florianópolis,v.21,n.4,p.15-19. 2008.
BRACKMANN, A.; FREITAS, S.T.; MELLO, A.M.; STEFFENS, C.A. Aplicação de 1-MCP em caqui 'quioto' armazenado sob refrigeração e atmosfera controlada. Revista Brasileira de Fruticultura, Jaboticabal, v.25, n.1, p.42-44, 2003.

ChitarRa, M. I. F.; CHITARRA, A. B. Póscolheita de frutos e hortaliças: fisiologia e manuseio. Lavras: Ed UFLA, 2005. 785 p.

CAMPO-DALL'ORTO, F. A.; OJIMA, M.; BARBOSA, W.; ZULlO, M. A. T. Novo processo de avaliação da adstringência dos frutos no melhoramento do caquizeiro. Bragantia, Campinas, v. 55, n. 2, p. 237-243, 1996.

DAOOD, H. G.; BIACS, P.; CZINKOTAI, B.; HOSCHKE, A. Chromatographic investigation of carotenoids, sugar and organic acids from Diospyros kaki fruits. Food Chemistry, London , v.45, p. 151-155, 1992.

DONAZZOLO, J.; BRACKMANN, A. Efeito do $\mathrm{CO}_{2}$ em atmosfera controlada na qualidade de caqui (diospyros kaki, l.) Cv. Fuyu. Revista Brasileira Agrociência, Pelotas, v. 8, n. 3, p. 241-245, set. dez, 2002.

EDAGI, F.K.; KLUGE, R. A. Remoção de adstringência de caqui: um enfoque bioquímico, fisiológico e tecnológico. Ciência Rural, Santa Maria, v.39, n.2, p.585-594, 2009.

FAGUNDES, A. F. Avaliação de diferentes doses de aminoetoxivinilglicina e substâncias inibidoras do escurecimento aplicadas por imersão póscolheita em frutos de caqui (Diospyros kaki L). 2004. 46 f. Dissertação (Mestrado em Agronomia)Universidade Estadual de Ponta Grossa, Ponta Grossa, 2004. 
FINGER, F. L.; VIEIRA, G. Controle da perda póscolheita de água em produtos hortícolas. Viçosa: UFV, 2002. 29 p.

GOMES, F.P. Curso de estatística experimental. 12.ed. Piracicaba: Nobel, 1987. 467p.

HARDENBURG, R.E.; WATADA, A.E.;WANG, C.Y. The comercial storage of fruits, vegetables, and florists and nursery stocks. Washington: Department of Agriculture, Agricultural Research Service, 1986. 136p. (Agriculture Handbook, 66).

INSTITUTO ADOLFO LUTZ. Métodos físicoquímicos para análise de alimentos. São Paulo: Instituto Adolfo Lutz, 2008. p. 1.020.

KAFERSTEIN, F. K.; MOY, G. G. Public health aspects of food irradiation. Journal of Public Health Policy, South Burlington, v.14, n.2, p.149-163, 1993.

KOVACS, E.; DJEDJRO, G.A.; SASS, P. Changes in organic acids of fruits after different treatment. Acta Horticulturae, The Hague, n.368, p.251-261, 1994.

MAPA. Ministério da Agricultura, Pecuária e Abastecimento. Método de Tillmans modificado. Disponível em: <http://www.agricultura.gov.br $>$. Acesso em: 20 fev. 2011

MAXIE, E.C.; SOMMER, N.F.; MITCHELL, F.G. Chemical, economic, physical and physiological limitations to irradiation of fruits. In: INTERNACIONAL ATOMIC ENERGY AGENCY. Desinfestation of fruits by irradiation. Vienna: IAEA, 1971. p.93-100.
PROGRAMABRASILEIRODEMODERNIZAÇÃO DA HORTICULTURA. Normas de classificação do Caqui. São Paulo: CQH; CEAGESP, 2000. (Documentos, 22).

SENTER, S. D.; CHAPMAN, G. W.; FORBUS Jr, W. R.; PAYNE, J. A. Sugar and nonvolatile acid composition of persimmons during maturation. Journal of Food Science, Chicago, v. 56, n. 4, p. 989-991, 1991.

SILVA, J.M; SILVA, J.P.; SPOTO, M.H.F. Características físico-químicas de abacaxi submetido à tecnologia de radiação ionizante como método de conservação pós-colheita. Ciência e Tecnologia de Alimentos, Campinas, v.28 n.1, p.139-145. Campinas jan./mar. 2008.

TURK, R. The cold storage of persimmons (Diospyros kaki cv. fuyo) harvested at different maturities and the effect of different $\mathrm{CO}_{2}$ applications on fruit ripening. Acta Horticulturae, The Hague, n.343, p.190-194, 1993.

VIDRIH, R.; SIMCIC, M.; HRIBAR, J. Storing of persimmon fruit under controlled atmosphere conditions. In: INTERNATIONAL HORTICULTURAL CONGRESS, 23., 1990, Firenze. Abstracts... Firenze : ISHS, 1990. v.2, p.3312.

WILLS, R.H.H.; LEE, T.H.; GRAHAM, D.; McGLASSON, W.B.; HALL, E.G. Postharvest an introduction to the physiology and handling of fruit and vegetables. Kensington: New South Wales University Press, 1981. 161p. 